\title{
Correlações entre Aspectos da Memória e Aprendizagem em População Idosa
}

\section{Correlation between Aspects of Learning and Memory in Elderly Population}

http://dx.doi.org/10.5007/2178-4582.2013v47n1p133

\author{
Juliana F. Cecato e José Eduardo Martinelli \\ Faculdade de Medicina de Jundiai, Jundiai/SP, Brasil
}

Luana L. Bartholomeu, Patricia P. Ferreira, José Maria Montiel

e Daniel Bartholomeu

Centro Universitário Fundação Instituto de Ensino para Osasco, Osasco/SP, Brasil

\begin{abstract}
O processo de envelhecimento vem crescendo nos últimos anos e o idoso no Brasil ainda carrega o estereótipo de incapacidade e improdutividade. Estudos utilizam a memória a fim de avaliar o comprometimento de pacientes com suspeita de síndromes demenciais. A importância das avaliações cognitivas nos idosos por meio de tarefas de aprendizagem e evocação livre podem predizer quadros de demência. O objetivo desse estudo foi avaliar a capacidade de aprendizagem em idosos que frequentam uma Faculdade da Terceira Idade no município de Jundiaí e que são atendidos em um Instituto de Geriatria e Gerontologia nesta cidade. Foram avaliados 95 idosos, com idade igual ou superior a 60 anos, de ambos os sexos, por meio da Bateria de Memória para Idosos (Elder Memory Test). Os resultados mostraram diferenças estatisticamente significativas nos cinco itens da evocação tanto no grupo separado por gênero, idade e escolaridade.
\end{abstract}

Palavras-chave: Avaliação Psicológica; - Psicodiagnóstico - Idoso - Aprendizagem.
Aging process has grown in recent years and the elderly in Brazil still carries the stereotype of disability and nonproductiveness. Studies use memory to assess the impairment of patients suspected of dementia. The importance of cognitive assessments in elderly through learning tasks and free recall may predict dementia. The aim of this study was to evaluate the capabilities of learning of elderly who attends a College of the Third Age in Jundiai and who are treated at the Institute of Geriatrics and Gerontology in this city. Ninety five elderly people aged over 60 years, of both sexes, were evaluated through an Elder Memory Test. Participants were grouped according to age, educational levels whereas the test averages were compared by a statistical program. The results showed statistically significant differences on five items evoking both groups separated by gender, age and education.

Keywords: Psychological Assessment - Psychodiagnostic Elderly - Learning.

\section{Introdução}

O envelhecimento populacional é um processo que vem sendo amplamente estudado em todo o mundo (QUINN et al, 2008), inclusive nos países em desenvolvimento (FERREIRA et al., 2009; OLIVEIRA-CAMPOS et al., 2011). Isto porque o tema é considerado um desafio para as políticas públicas 
no sentido de preparar a sociedade para atender as consequências do envelhecimento (OLIVEIRA-CAMPOS et al, 2011). Na área da saúde, a preocupação com a longevidade está no fato de ser fator de risco para síndromes de demências, dentre as quais se pode citar as demências do tipo Alzheimer (DA), Corpúsculos de Lewy, Frontotemporais e Vascular (CARAMELLI et al., 2002; TEIXEIRA et al., 2008), sendo que a prevalência desses casos aumenta consideravelmente a partir dos 60 anos (TEIXEIRA et al., 2008).

No que tange ao senso comum, quando o assunto é demência, a tendência é a de um quadro de piora desta população em decorrência da falta de informações e, consequentemente, da pouca procura por atendimento médico, pois acredita-se, equivocadamente, que é normal o idoso esquecer (SILVA et al., 2002). Abreu et al (2005) referem-se aos esquecimentos como comprometimentos da memória que devem ser examinados, enquanto Charchat-Fichman et al (2005) relatam a importância de se investigar esse comprometimento o mais precocemente possível, a fim de permitir intervenções clínicas caso se confirme o diagnóstico de síndrome demencial.

A demência é uma síndrome clínica, manifestada por declínio da memória e, secundariamente, de outras funções corticais superiores, além de alterações comportamentais que também acompanham o quadro (LUDERS et al, 1996). A forma mais comum de estabelecer o diagnóstico de demência é feito pelo Manual Diagnóstico e Estatístico (DSM) adotado pela Associação de Psiquiatria Americana (American Psychiatric Association - APA), em sua $4^{\mathrm{a}}$ edição (1994). O DSM-IV correlaciona-se com a Classificação Internacional das Doenças, em sua $10^{\mathrm{a}}$ edição (CID-10), desenvolvida pela Organização Mundial de Saúde (OMS, 1992). É importante salientar que esses critérios diagnósticos são responsáveis por fornecer um diagnóstico provável da DA. O diagnóstico definitivo é realizado apenas com exame anatomopatológico, ou seja, esse exame só é possível com amostra de tecido cerebral retirado do paciente após o óbito (LUDERS et al, 1996).

$\mathrm{O}$ fator de risco mais importante para a DA é a idade avançada. Contudo, traumatismos cranioencefálicos repetidos também podem contribuir com esse quadro (DAMASCENO, 2006). Poucos estudos sugerem fatores genéticos. O que se sabe até o momento é que a proteína $\beta$-amilóide, após sofrer um processo de mutação, está associada ao desenvolvimento da doença. Outro fator genético muito estudado é a Apolipoproteína E (ApoE), que sugere aumento de risco de $50 \%$ para DA quando encontrados grandes quantidades do alelo ApoE 4 (DAMASCENO, 2006). Porém, os estudos com o alelo ApoE 4 não são considerados marcadores biológicos para DA. Uma explicação para esse fato é que em estudos realizados com portadores de DA, em muitos pacientes não foram encontrados este alelo (DAMASCENO, 2006). Damasceno (2006) acredita que possam existir ainda outros fatores genéticos, associados a fatores ambientais, que ainda não foram identificados como os responsáveis pelo desenvolvimento da doença.

A investigação diagnóstica das síndromes demenciais se baseia em uma série de exames, dentro dos quais podem-se citar os exames clínicos, neurop- 
sicológicos, laboratoriais e de neuroimagem para se estabelecer o diagnóstico diferencial (CARAMELLI et al, 2002). No caso de idosos com doença de Alzheimer, o processo neurodegenerativo se inicia com a deterioração da região cerebral denominada hipocampo, com posterior comprometimento de regiões corticais (CARAMELLI et al, 2002; TEIXEIRA et al, 2008). As avaliações neuropsicológicas fornecem importantes informações sobre dados cognitivos mais específicos do que apenas os exames de neuroimagem. Bodi et al. (2009) acreditam que as avaliações da aprendizagem podem evidenciar fases prodrômicas da DA. Em seus resultados, os pacientes com DA leve conseguiram promover uma aprendizagem associativa de tarefas com cartas, fazendo associações da aprendizagem adquirida semelhantes aos idosos-controle. O que diferenciou os dois grupos foi que, quando houve a necessidade de se fazer generalizações com o material aprendido com as mesmas cartas, os pacientes com DA leve tiveram maior comprometimento. Estudos referem-se ao declínio da memória recente como os primeiros sinais de demência (ALMEIDA, 1998; CHEN et al, 2011; YASSUDA, 2002) e, para melhor auxiliar o diagnóstico clínico, Bennett et al. (2002) apontam que a piora dos idosos em tarefas que avaliam a aprendizagem pode ser considerado um fator preditivo para o diagnóstico de DA. Esse fato pode ser elucidado com base no funcionamento do executivo central. $\mathrm{O}$ executivo central é parte controladora da memória operacional, responsável pelas estratégias de codificação das informações novas e seu eventual armazenamento. O comprometimento do executivo central, observado nas tarefas de aprendizagem, pode indicar uma síndrome demencial em evolução (BENNETT et al, 2002).

$\mathrm{O}$ processo de aprendizagem no idoso envolve diversos fatores, como a memória, inteligência, atenção e funções sensoriais (PERLMUTTER et al, 1992). A capacidade de aprendizagem do idoso não se altera e pode-se observar no envelhecimento normal que as funções intelectuais se mantêm por toda a vida (TAVARES et al, 2002). Outro ponto importante de se considerar é que a capacidade de aprendizagem está diretamente ligada à atenção, concentração, motivação, interesse e ausência de doença. Isso significa dizer que para ocorrer aprendizagem no idoso sem comprometimento cognitivo é necessário que a atividade seja de interesse para sua vida e que o motive (TAVARES et al, 2002). O estudo da aprendizagem no idoso vem tendo destaque e espaço no meio científico e o seu declínio pode ser considerado como um marcador para o declínio cognitivo patológico. Sterlin et al. (2008) analisaram as regiões hipocampais de idosos com diagnóstico de DA, Comprometimento Cognitivo Leve (CCL) e de idosos normais (sem declínio cognitivo). Os pesquisadores utilizaram os critérios diagnósticos de Petersen (2001 apud STERLIN et al, 2008) para estabelecer o CCL. Para as análises do hipocampo, os autores utilizaram a ressonância magnética (RM) durante tarefas de aprendizagem a fim de verificar a dinâmica das respostas neuronais durante a tarefa proposta. Encontraram no grupo composto por idosos normais que a região do hipocampo apresentava grande dinâmica neuronal durante as tarefas de aprendizagem. $\mathrm{O}$ mesmo resultado não ocorreu com o grupo com diagnóstico de CCL e com o grupo formado por pacientes com DA. 
Considerando a investigação diagnóstica, bem como para elucidar e mensurar a capacidade cognitiva dos idosos - mesmo daqueles que apresentam comprometimento mental - tal temática torna-se importante para diversos profissionais de saúde, especialmente no que diz respeito ao desenvolvimento de programas de reabilitação e estimulação cognitiva (YASSUDA et al, 2006). Nesse contexto, as discussões na área das avaliações psicológicas se tornam fundamentais para buscar ferramentas que avaliem o grau de comprometimento e gravidade que se apresentam em determinados transtornos (CAMPOS, 2008). Em outro apontamento, devido ao aumento da expectativa de vida, à escassez de informações sobre dados brasileiros sobre aprendizagem nos idosos e pela importância da manutenção da autonomia e qualidade de vida dos idosos, o projeto de avaliação da aprendizagem no envelhecimento se torna relevante para a área da saúde e da gerontologia.

Desta maneira, este estudo teve como objetivo avaliar, por meio da Bateria de Memória para Idosos (Elder Memory Test, em preparação), a capacidade de aprendizagem em idosos residentes em um município do interior do estado de São Paulo que frequentam uma Faculdade da Terceira Idade e que são atendidos no Instituto de Geriatria e Gerontologia. Ou seja, visa-se descrever possíveis correlações no desempenho dos testes de memória recente e tardia com a aprendizagem, além de avaliar o desempenho da aprendizagem em relação ao gênero.

\section{Metodologia}

\section{Os participantes}

Foram avaliados 95 idosos que vivem na comunidade com idade igual ou superior a 60 anos, de ambos os sexos, que frequentam a faculdade aberta da Terceira Idade e que são atendidos em um Instituto de Geriatria e Gerontologia, numa cidade do interior do estado de São Paulo. Os idosos participaram da pesquisa por livre e espontânea vontade, de acordo com a assinatura dos termos de consentimento ${ }^{1}$.

\section{O instrumento: Elder Memory Test}

Para a avaliação da memória, foram utilizados slides em que 20 figuras foram apresentas com um tempo de memorização de 2 minutos. Logo após a exibição das figuras, pediu-se para que os participantes escrevessem na folha de respostas as figuras que se lembraram de ter sido apresentadas. Em seguida, foi apresentado novamente, com um tempo de 2 minutos para a memorização, 20 figuras. Em seguida, foi apresentado um distrator com duração de 1 minuto e 30 segundos. Após a realização da tarefa, pediu-se

1 Este projeto teve aprovação do Comitê de Ética sob o número 099/2010 do Comitê de Ética em Pesquisa (CEP) da Anhanguera Educacional. 
para os participantes evocarem o que lembraram das 20 figuras. Novamente, foram exibidas as figuras durante 2 minutos para a memorização. Os distratores foram apresentados em seguida, agora aumentando-se o tempo, ou seja, distratores com 2 e 3 minutos, após a exibição das 20 figuras. Depois do último distrator (de 3 minutos), as 20 figuras foram novamente exibidas para a memorização, desta vez também com um tempo de 2 minutos. A evocação só foi feita após um tempo mínimo de 15 minutos, a fim de avaliar a memória tardia.

Com dissemos, esta prova (Elder Memory Test, em preparação) consiste na apresentação de figuras, dentre as quais estão presentes frutas, animais, objetos do cotidiano (como xícara, abajur, etc) e meios de transporte. A avaliação iniciou-se com a apresentação, pela examinadora, de uma prancha com as 20 figuras, tendo sido solicitado que os participantes tentassem memorizá-las. Eles foram avisados de que teriam 2 minutos para essa atividade de memorização. Terminado o tempo, os participantes deveriam evocar as figuras que conseguiram memorizar, não havendo necessidade de respeito a ordem em que foram apresentadas. Esse procedimento foi repetido 5 vezes (E1, E2, E3, E4 e E5), com intervalos nos quais foram apresentados distratores, com exceção do intervalo E1 em que foi exigida a evocação imediata das figuras. Os intervalos têm duração de 1 minuto e 30 segundos, entre E1 e E2; 2 minutos, entre E2 e E3; 3 minutos, entre E3 e E4; e 15 minutos entre E4 e E5. Ao final de cada evocação, a prancha com as 20 figuras foi apresentada por 2 minutos.

\section{Análise dos dados}

A análise de dados foi feita por meio do programa SAS System para Windows (Statistical Analysis System) versão 6.12 e SPSS 15.0 (2007), onde foram efetuadas estatísticas descritivas quanto à distribuição da amostra (idade, gênero, escolaridade). As diferenças significativas entre os grupos de idade e escolaridade e os escores do teste de memória foram feitas pela análise ANOVA (post hoc), estabelecendo-se um nível de significância de 5\%. Foram obtidos ainda coeficientes de correlação de Pearson (r), entre os escores da aprendizagem e as variáveis escolaridade e idade, tentando-se identificar a influência dessas variáveis nos resultados. Como o teste aplicado para avaliar a aprendizagem ainda está em preparação, foi realizada a análise estatística de Kolmogorov-Smirnov (KS) para verificar se o teste corresponde a uma distribuição normal. Segundo os resultados observados, a amostra corresponde a uma distribuição não-paramétrica. Foi utilizado o teste de Mann-Whitney a fim de verificar diferenças significativas entre os escores da aprendizagem e os grupos de escolaridade. O teste de Kruskal -Wallis foi utilizado para diferenciar a aprendizagem em relação aos grupos de gênero e idade. Para o cálculo da aprendizagem foram consideradas as respostas dadas em E1 e E5, ou seja, o valor da aprendizagem foi calculado subtraindo-se a pontuação da última e da primeira evocação (E5-E1). 


\section{Resultados}

Quanto ao gênero, do total de participantes, $79(83,16 \%)$ eram do sexo feminino. Em relação à escolaridade, 25 idosos (26,32\%) tinham de 1 a 4 anos de estudo; 14 (14,74\%) de 5 a 8 anos; sendo que a maioria (58,94\%), ou seja, os 56 idosos restantes tinham alta escolaridade, 9 anos de estudo ou mais. A média de idade encontrada foi igual a 67,30 (desvio padrão [SD] = 6,02 , mínimo $=60$, máximo $=91$, Mediana $=66$ ). A análise estatística Kolmogorov-Smirnov, para verificar a distribuição da amostra, pode ser observada na Tabela 1. Comprova-se nesta tabela que a amostra segue uma distribuição normal nos testes E4 e E5 ( $>>0,05)$.

Tabela 1. Análise do teste Kolmogorov-Smirnov comprovando que a amostra segue uma distribuição normal. $\mathrm{n}=$ número de participantes, $\mathrm{sd}=$ desvio padrão.

\begin{tabular}{lccccc}
\hline Amostra & $\boldsymbol{E} 1$ & $\boldsymbol{E 2}$ & $\boldsymbol{E 3}$ & $\boldsymbol{E 4}$ & $E 5$ \\
\hline$N$ & 95 & 95 & 95 & 95 & 95 \\
Média & 11,42 & 12,28 & 14,87 & 16,12 & 17,05 \\
$S D$ & 2,60 & 3,08 & 2,57 & 2,37 & 2,41 \\
$P$ & 0,206 & 0,096 & 0,080 & $\mathbf{0 , 0 2 2}$ & $\mathbf{0 , 0 3 0}$ \\
\hline
\end{tabular}

Fonte: Tabela elaborada pelos autores

Foram feitas análises comparativas entre as variáveis categóricas: gênero, idade e escolaridade e as evocações do teste. Pode-se observar que as mulheres apresentam médias maiores em todos os testes (E1, E2, E3, E4 e E5), como verifica-se na Tabela 2. Um dado importante observado foi que houve diferenças estatisticamente significativas entre o gênero no E3 $(p=0,003), E 4(p=0,019)$ e $E 5(p=0,042)$. Com relação à variável idade, pode ser evidenciado que os idosos mais jovens (grupo com idade entre 60 a 69 anos) apresentam médias menores no teste E1 e E2, mas médias superiores nos demais testes (Tabela 3$)$. Verificam-se diferenças estatisticamente significativas apenas no E3 $(p=0,040)$ e $E 5(p=0,021)$ entre os grupos separados por idade. Os níveis de escolaridade foram divididos em 3 grupos (de 1 a 4 anos; de 5 a 8 anos; e acima de 9 anos), tendo sido observadas médias inferiores nos participantes com menos tempo de escolaridade ( 1 a 4 anos) em todos os testes (Tabela 4). A partir desse dado, pode-se inferir que os participantes com mais anos de estudo tiveram pontuação superior quando comparados ao grupo de idosos com poucos anos de estudo. As diferenças entre os grupos mostram-se significativas em relação ao $E 2(p=0,012), E 3$ $(p=0,017)$ e $E 4(p=0,013)$. 
Tabela 2. Comparação das evocações dos participantes em relação a variável categórica do gênero. $\mathrm{n}=$ número de participantes, $\mathrm{sd}=$ desvio padrão.

*Mann-Whitney.

\begin{tabular}{ccccccc}
\hline Testes & Grupo & $\boldsymbol{N}$ & Média & $\boldsymbol{S D}$ & $\begin{array}{c}\text { Variação } \\
\text { (min-máx) }\end{array}$ & $* \boldsymbol{*}$ \\
\hline \multirow{2}{*}{ E1 } & $F$ & 79 & 11,48 & 2,53 & $4-16$ & 0,670 \\
& $M$ & 16 & 11,12 & 3,01 & $6-9$ & \\
\multirow{2}{*}{ E2 } & $F$ & 79 & 12,40 & 2,88 & $6-19$ & 0,760 \\
& $M$ & 16 & 11,69 & 4,01 & $0-17$ & \\
\multirow{2}{*}{ 33 } & $F$ & 79 & 15,19 & 2,53 & $5-20$ & $\mathbf{0 , 0 0 3}$ \\
& $M$ & 16 & 13,31 & 2,24 & $9-18$ & \\
$E 4$ & $F$ & 79 & 16,35 & 2,33 & $10-20$ & $\mathbf{0 , 0 1 9}$ \\
& $M$ & 16 & 14,94 & 2,29 & $11-20$ & \\
E5 & $F$ & 79 & 17,29 & 2,29 & $10-20$ & $\mathbf{0 , 0 4 2}$ \\
& $M$ & 16 & 15,87 & 2,68 & $9-20$ & \\
\hline
\end{tabular}

Fonte: Tabela elaborada pelos autores

Tabela 3. Comparação das evocações dos participantes em relação a variável categórica da idade. $\mathrm{n}=$ número de participantes, $\mathrm{sd}=$ desvio padrão.

*Mann-Whitney

\begin{tabular}{ccccccc}
\hline \multirow{2}{*}{ Testes } & Grupo & $\boldsymbol{N}$ & Média & SD & $\begin{array}{c}\text { Variação } \\
\text { (min-máx) }\end{array}$ & $* \boldsymbol{*}$ \\
\hline \multirow{2}{*}{ E1 } & 60 a 69 anos & 64 & 12,27 & 3,28 & $0-19$ & 0,997 \\
& $>70$ anos & 31 & 12,32 & 2,68 & $7-19$ & \\
\multirow{2}{*}{ E2 } & 60 a 69 anos & 64 & 11,39 & 2,73 & $4-16$ & 0,946 \\
& $>70$ anos & 31 & 11,48 & 2,35 & $7-16$ & \\
\multirow{2}{*}{ E3 } & 60 a 69 anos & 64 & 15,16 & 2,73 & $5-20$ & $\mathbf{0 , 0 4 0}$ \\
& $>70$ anos & 31 & 14,29 & 2,13 & $10-18$ & \\
\multirow{2}{*}{ E4 } & 60 a 69 anos & 64 & 16,41 & 2,27 & $10-20$ & 0,079 \\
& $>70$ anos & 31 & 15,52 & 2,50 & $11-20$ & \\
\multirow{2}{*}{ E5 } & 60 a 69 anos & 64 & 17,37 & 2,45 & $9-20$ & $\mathbf{0 , 0 2 1}$ \\
& $>70$ anos & 31 & 16,39 & 2,20 & $11-20$ & \\
\hline
\end{tabular}


Tabela 4. Comparação das evocações dos participantes em relação a variável categórica da escolaridade. $\mathrm{n}=$ número de participantes, $\mathrm{sd}=$ desvio padrão.

*Kruskal-Wallis.

\begin{tabular}{|c|c|c|c|c|c|c|}
\hline Testes & Grupo & $N$ & Média & $S D$ & $\begin{array}{c}\text { Variação } \\
\text { (min-máx) }\end{array}$ & $* p$ \\
\hline \multirow{3}{*}{ E1 } & 1 a 4 anos & 25 & 10,60 & 2,20 & $6-14$ & \multirow{3}{*}{0,118} \\
\hline & 5 a 8 anos & 14 & 11,71 & 2,76 & $8-16$ & \\
\hline & $>9$ anos & 56 & 11,71 & 2,69 & $4-16$ & \\
\hline \multirow{3}{*}{$E 2$} & 1 a 4 anos & 25 & 11,04 & 2,57 & $6-16$ & \multirow{3}{*}{0,012} \\
\hline & 5 a 8 anos & 14 & 11,43 & 4,40 & $0-17$ & \\
\hline & $>9$ anos & 56 & 13,05 & 2,70 & $7-19$ & \\
\hline \multirow{3}{*}{ E3 } & 1 a 4 anos & 25 & 13,76 & 2,98 & $5-17$ & \multirow{3}{*}{0,017} \\
\hline & 5 a 8 anos & 14 & 14,07 & 3,29 & $10-20$ & \\
\hline & $>9$ anos & 56 & 15,57 & 1,90 & $11-20$ & \\
\hline \multirow{3}{*}{$E 4$} & 1 a 4 anos & 25 & 15,00 & 2,71 & $10-20$ & \multirow{3}{*}{0,013} \\
\hline & 5 a 8 anos & 14 & 15,64 & 2,06 & $12-19$ & \\
\hline & $>9$ anos & 56 & 16,73 & 2,09 & $12-20$ & \\
\hline \multirow{3}{*}{ E5 } & 1 a 4 anos & 25 & 15,96 & 3,10 & $9-20$ & \multirow{3}{*}{0,136} \\
\hline & 5 a 8 anos & 14 & 17,36 & 2,34 & $14-20$ & \\
\hline & $>9$ anos & 56 & 17,46 & 1,92 & $12-20$ & \\
\hline
\end{tabular}

Fonte: Tabela elaborada pelos autores

Também foi realizado um teste de correlação entre as variáveis categóricas (idade, gênero e escolaridade) e as evocações do teste de memória. Podese observar, na tabela 5, correlações significativas, fracas e negativas no E3 $(r=-0,27 ; p=0,007), E 4(r=-0,22 ; p=0,028)$ e $E 5(r=-0,22 ; p=0,031)$ com relação ao gênero. Coeficientes de correlações significativas, fracas e positivas podem ser observadas em relação à variável escolaridade nos testes E2 $(r=0,29 ; p=0,004)$ e $E 5(r=0,26 ; p=0,012)$, e um valor de correlação significativo e moderado no teste $\mathrm{E} 3(\mathrm{r}=0,32 ; \mathrm{p}=0,002)$ e $\mathrm{E} 4(\mathrm{r}=0,32 ; \mathrm{p}=0,001)$. 
Tabela 5. Análises de correlação entre as variáveis categóricas e os testes de memória. $* r=$ correlação de Pearson; $\mathrm{p}=\mathrm{x}^{2}$.

\begin{tabular}{ccccccc}
\hline Variáveis & & E1 & E2 & E3 & E4 & E5 \\
\hline \multirow{2}{*}{ Gênero } & $r$ & $-0,05$ & $-0,09$ & $\mathbf{- 0 , 2 7}$ & $\mathbf{- 0 , 2 2}$ & $\mathbf{- 0 , 2 2}$ \\
& $p$ & 0,620 & 0,399 & $\mathbf{0 , 0 0 7}$ & $\mathbf{0 , 0 2 8}$ & $\mathbf{0 , 0 3 1}$ \\
\multirow{2}{*}{ Idade } & $r$ & 0,01 & 0,01 & $-0,16$ & 0,18 & $-0,19$ \\
& $p$ & 0,871 & 0,933 & 0,124 & 0,086 & 0,060 \\
& $r$ & 0,17 & $\mathbf{0 , 2 9}$ & $\mathbf{0 , 3 2}$ & $\mathbf{0 , 3 2}$ & $\mathbf{0 , 2 6}$ \\
Escolaridade & & 0,091 & $\mathbf{0 , 0 0 4}$ & $\mathbf{0 , 0 0 2}$ & $\mathbf{0 , 0 0 1}$ & $\mathbf{0 , 0 1 2}$ \\
\hline
\end{tabular}

Fonte: Tabela elaborada pelos autores

O cálculo da aprendizagem foi feito subtraindo-se os escores de E5 dos de E1. As médias entre os grupos são descritas na Tabela 6. Não foram encontradas diferenças significativas entre as variáveis gênero e escolaridade. Contudo, observa-se uma diferença estatisticamente significativa quando se divide o grupo pela idade $(\mathrm{p}=0,0032)$. Na Tabela 6 , verifica-se que a média do grupo entre 60 a 69 anos foi igual a 5,10 e que o grupo acima de 70 anos obteve uma média inferior ou igual a 4,07. Como a escolaridade foi a única variável dividida em 3 grupos, optou-se por analisar os grupos pelos níveis de escolaridade, ou seja, grupo de 1 a 4 anos com o grupo de 5 a 8 anos e acima de 9 anos, e 5 a 8 anos com o grupo > de 9 anos. Separando a variável escolaridade, evidencia-se uma diferença significativa quando se compara o grupo de participantes de 1 a 4 anos com o grupo acima de 9 anos. Por meio do teste de Komogorov-Smirnov verifica-se que a subtração E5 menos E1 trata-se de uma análise paramétrica e, por essa razão, foi escolhido o teste t de Student para analisar os grupos. Como era esperado, o teste estatístico mostrou diferença significativa quando dividiu-se os grupos pelos níveis de escolaridade $(\mathrm{p}<0,0001)$, como descrito na Tabela 6 .

A escolaridade foi a variável que apresentou maior diferença entre os grupos em relação à aprendizagem, como mostram os resultados obtidos com a subtração E5-E1 (Gráfico 1). Verifica-se o ganho maior dos participantes com mais de 9 anos de estudo na comparação com os outros grupos. O ganho obtido nesse grupo chega até os 15 pontos, sendo que o máximo de 10 pontos foi alcançado pelo grupo de 1 a 4 anos; e o de 5 a 8 anos foi igual a 10 pontos. 
Tabela 6. Cálculo da aprendizagem (e5 - e1) em relação aos grupos. *Mann -whitney; **t Student.

\begin{tabular}{|c|c|c|c|}
\hline Testes & $\begin{array}{c}\text { Resultados } \\
\text { (Média E5 - Média E1) }\end{array}$ & $* p$ & **Por grupos \\
\hline \multicolumn{4}{|l|}{ Gênero } \\
\hline Feminino & 5,81 & & \\
\hline Masculino & 4,75 & 0,127 & \\
\hline \multicolumn{4}{|l|}{ Idade } \\
\hline 60 a 69 anos & 5,10 & & \\
\hline$>70$ anos & 4,07 & $\mathbf{0 , 0 3 2}$ & \\
\hline \multicolumn{4}{|l|}{ Escolaridade } \\
\hline 1 a 4 anos & 5,36 & & 0,0001 \\
\hline 5 a 8 anos & 5,65 & & 0,0001 \\
\hline$>9$ anos & 5,75 & 0,837 & 0,0001 \\
\hline
\end{tabular}

Fonte: Tabela elaborada pelos autores

Gráfico 1. Representação gráfica da pontuação nos testes de evocação (E5-E1) entre os participantes idosos separados por níveis de escolaridade.

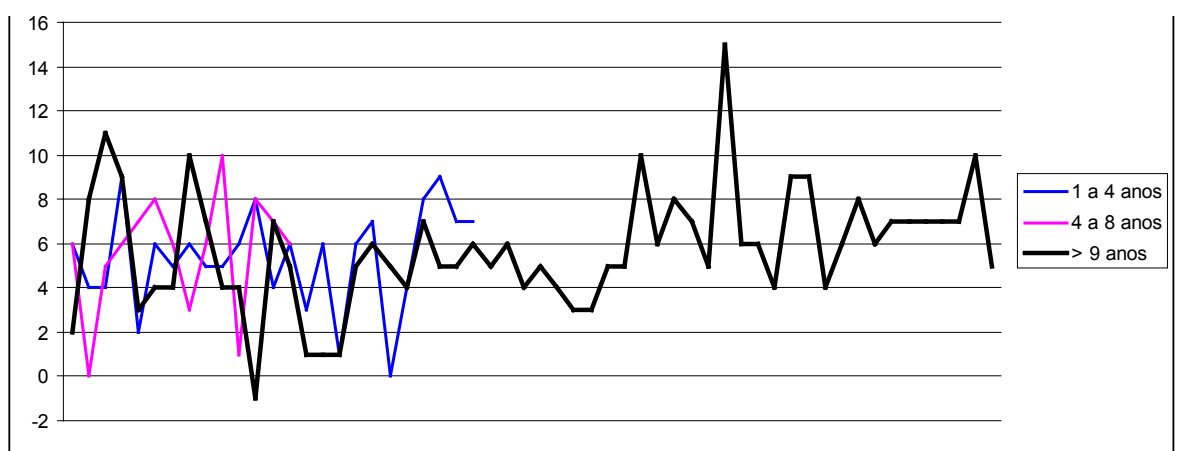

Fonte: Gráfico elaborado pelos autores 


\section{Discussão}

A incessante busca para encontrar um marcador biológico para o envelhecimento é uma questão antiga (DAMASCENO, 2006). Assim, tentar encontrar um fator biológico para a velhice não é uma questão simples, especialmente no atributo aprendizagem. Pesquisas utilizando uma parte do cromossomo buscam compreender os efeitos do envelhecimento. O telômero é a região final do cromossomo, responsável pela divisão celular. Em cada divisão celular o telômero fica mais curto e chega até um ponto onde não é mais capaz de promover essa divisão e, consequentemente, a célula também deixa de existir. Esse fato está ligado ao envelhecimento, pois em pessoas idosas o telômero é mais curto quando comparado a adultos jovens (EPEL, 2009; EPEL et al, 2008).

Contudo, as pesquisas de Epel (2009; et al, 2008) apontam que o encurtamento dos telômeros está mais ligado a fatores estressantes durante a vida dos indivíduos do que a marcadores do envelhecimento. Isso significa dizer que, durante o curso de vida, se o indivíduo passou por mais situações estressantes que outra pessoa de mesma idade, terá o telômero mais curto quando chegar na velhice. O telômero parece ser apenas mais um dos diversos fatores envolvidos no processo de envelhecimento. Em outros apontamentos em relação à busca de compreensão dos fatores envolvidos na velhice, Damasceno (2006) relata a função das proteínas mutagênicas de $\beta$-amilóide que se depositam entre as conexões sinápticas envolvidas na doença. Mas o que deve ser avaliado é como ocorre esse processo mutagênico, isto é, o que acontece com o organismo que o faz produzir esse tipo de proteína geradora do quadro demencial. A ApoE também é outro fator biológico muito pesquisado na DA. A presença do alelo ApoE 4 sugere um risco de até 50\% em se desenvolver a doença. Mas mesmo idosos que apresentem esse alelo podem não chegar a desenvolver o quadro demencial (DAMASCENO, 2006).

As avaliações neuropsicológicas fornecem importantes informações sobre dados cognitivos mais específicos do que apenas os exames de neuroimagem. Bodi et al al. (2009) acreditam que as avaliações da aprendizagem podem predizer fases prodrômicas da DA. Em seus resultados, os pacientes com DA leve conseguiram promover uma aprendizagem associativa de tarefas com cartas, fazendo associações da aprendizagem adquirida semelhantes aos dos idosos-controle. O que diferenciou os dois grupos foi que, quando houve a necessidade de se fazer generalizações com o material aprendido com as mesmas cartas, os pacientes com DA leve tiveram maior comprometimento. Sterlin et al (2008) pesquisaram 33 idosos, sendo 12 normais (sem queixas cognitivas) e $21 \mathrm{com}$ comprometimento de aprendizagem e memória. Os pesquisadores observaram que tarefas de aprendizagem simples podem acontecer mesmo sem o envolvimento do hipocampo. Isso significa dizer que pacientes com DA, geralmente com comprometimento da região hipocampal, serão capazes de assimilar tarefas que envolvam aprendizagens simples. 
Esses achados corroboram os dados observados nesta pesquisa. $\mathrm{O}$ grupo com DA conseguiu aumentar o número de figuras evocadas, observados nos momentos 3 e 4 do Gráfico 1. Esse fato pode também explicar os resultados encontrados na Tabela 3, com o cálculo de aprendizagem. Era esperado que os pacientes com diagnóstico de DA não conseguissem aprender nenhuma figura no momento final (E5). Contudo, pôde-se observar que 2 pacientes conseguiram aumentar uma figura verbalizada, e 2 pacientes mantiveram o mesmo número de figuras no seu repertório depois do período de tempo determinado pela tarefa. Apenas 2 pacientes obtiveram um número negativo. $\mathrm{O}$ aumento de figuras verbalizadas e a manutenção desse número pode ser explicado pelo trabalho desses autores em que a aprendizagem simples é possível, pois o hipocampo não é utilizado para essa tarefa.

Já os dois pacientes que obtiveram números negativos podem estar mais comprometidos com relação à doença, ou seja, quanto maior a pontuação da escala CDR (Clinical Dementia Rating) em que o paciente se encontra maior comprometimento hipocampal vai apresentar (HUGHES et al, 1982; MAIA et al, 2006; CHAVES et al, 2007). Pacientes com grau de demência mais grave apresentam maior comprometimento cognitivo, maior dependência nas AVDs e maior atrofia cerebral quando comparado a idosos normais (HUGHES et al, 1982). A hipótese para piora dos dois pacientes está no fato de poderem apresentar nível mais elevado de comprometimento com relação aos outros 4 idosos que preencheram os critérios para o grupo controle. É importante avaliar o quanto pacientes com DA conseguem adquirir e que tipo de aprendizagem pode ser assimilado devido ao papel da reabilitação cognitiva. Estudos apontam a capacidade de novas comunicações sinápticas no cérebro, o que denomina-se de plasticidade neuronal (PRESTES, 1998). Saber qual ponto do cérebro que está comprometido pode ser fundamental para melhorar o comportamento indesejável (COSENZA et al, 2008). Estudos com reabilitação cognitiva mostram a importância da aprendizagem para as mudanças comportamentais em pacientes que tiveram algum comprometimento cerebral (COSENZA et al, 2008; PONTES et al, 2008) e são fundamentais para recuperar regiões cerebrais danificadas (MACEDO et al, 2008).

A importância do trabalho com reabilitação neuropsicológica não está apenas no profissional de saúde, mas também no ambiente estimulador, como o que o paciente vai encontrar em seu ambiente familiar (BONINI, 1998). As melhoras cognitivas e comportamentais dos processos de reabilitação são demoradas, o processo é longo e os treinamentos devem se aproximar o mais possível da singular vida prática do indivíduo (ANDRADE, 2008). No estudo de Provencher et al (2009) trabalhou-se a reabilitação das AVDs com uma paciente de 77 anos e com diagnóstico de DA. Após nove meses do fim das intervenções observou-se que a paciente ainda participava do grupo de orações e respondia a um estímulo sonoro que a fazia lembrar de determinada obrigação. O relato de caso, expresso por Provencher et al (2009), também está de acordo com os achados desta pesquisa, onde verificou-se que os pacientes 
com DA leve conseguem promover uma aprendizagem simples, mesmo com comprometimento degenerativo. Os resultados do grupo-controle evidenciam que a capacidade de aquisição das informações aumenta à medida em que a aprendizagem ocorre (Gráfico 1). Esse dado ajuda a entender que o processo natural do envelhecimento não ocorre com perdas cognitivas, pelo contrário, o idoso normal tem boa memória e é capaz de aprender novas informações (ÁVILA et al, 2008; NERI, 2007). Se essas dificuldades estão presentes, é possível que indiquem uma patologia, como um processo neurodegenerativo (NERI, 2007; TEIXEIRA et al, 2008). Sinais de perda de memória ou de falta de atenção devem ser investigados, pois a confirmação do diagnóstico irá auxiliar no tratamento do paciente e na conduta do familiar diante de tais problemas.

\section{Considerações finais}

O presente estudo objetivou descrever possíveis correlações no desempenho dos testes de memória recente e tardia (Elder memory Test) com a aprendizagem e avaliar o desempenho da aprendizagem dos idosos em relação ao gênero. Neste sentido é importante descrever a importância do psicólogo na área da saúde e da avaliação neuropsicológica, como diagnóstico diferencial, em pacientes com quadros de demência. Os resultados evidenciaram que não é pelo fato de um individuo e/ou paciente ser idoso que se deve acreditar que os esquecimentos são próprios do processo de envelhecimento. Pelo contrário, se há queixa do paciente ou do familiar, deve-se levar em consideração essas queixas com mais profundidade, de modo a compreender os processos envolvidos nessas manifestações. Neste sentido, cabe mencionar que esquecimentos frequentes como, por exemplo, onde guardam os seus pertences pessoais ou lembrar e/ou contar estórias antigas constantemente podem ser sinais de que as funções cerebrais estejam alteradas. Outros fator que deve ser considerado são as alterações comportamentais, pois tendem a surgir com o avanço do quadro demencial, especialmente na doença de Alzheimer, tais como irritabilidade, insônia, labilidade emocional, dentre outros.

De acordo com os procedimentos adotados neste estudo, a avaliação neuropsicológica demonstrou ser um procedimento eficiente no trabalho de investigação das demências, especialmente em instituições geriátricas, e também que as avaliações devem ser direcionadas a um público-alvo, levando em consideração a idade e os anos de escolaridade dos indivíduos, principalmente dos pacientes idosos. Neste sentido, é importante salientar que a relação terapeuta-paciente é fundamental para o desenvolvimento do trabalho de identificação na área da saúde; e que, principalmente, não se deve infantilizar o idoso, que deve ser tratado com respeito pelos anos de experiência que a vida lhe proporcionou. Mesmo nos casos de idosos com demência, torna-se importante entender os comportamentos bizarros para, posteriormente, explicar aos familiares e cuidadores as fases da doença, os sintomas presentes em cada 
individuo, as alterações comportamentais e cognitivas que a doença ainda vai causar, e quais os procedimentos que serão adotados pelos profissionais envolvidos.

Seguindo os apontamentos anteriormente descritos, pode-se concluir que o trabalho realizado com idosos, especialmente com quadros demência, além de enriquecedor para as práticas clínicas na área da saúde é extremamente importante nesta fase da vida como meio de facilitar o desenvolvimento de estratégias de intervenção. Um outro ponto de extrema relevância diz respeito ao desenvolvimento de estratégias facilitadoras para os familiares de tais pacientes, uma vez que a não compreensão de quadros demência transforma-se em elemento dificultador para a melhora sintomatológica e para a qualidade de vida de tais pacientes. No que se refere às avaliações, estas devem considerar um aspecto de suma importância: o nível de escolaridade do indivíduo. Esta pesquisa corroborou o que outros estudos descrevem, isto é, que existe um ganho maior de procedimentos de intervenção em sujeitos com mais de 9 anos de escolaridade quando comparados aos de outros grupos com menor escolarização. Os ganhos e benefícios obtidos podem ser descritos como aproximadamente de $15 \%$ a mais do que o de outros grupos. Em outras populações clínicas, este índice pode ser avaliado como sendo baixo, porém para esta população representa um valor significativo. Ou seja, o grau de escolaridade deve ser considerado nos procedimentos de avaliação e intervenção, especialmente psicológica.

Finalmente, devem ser considerados outros aspectos de suma importância na avaliação desta população, tais como inserção social e o próprio contexto familiar. Estes fatores merecem especial atenção, por servirem de respaldo aos procedimentos a serem adotados. Neste sentido, novas pesquisas devem ser realizadas a fim de confirmar os achados deste estudo. A investigação com um maior numero de participantes se faz necessária para elucidar outros achados relevantes e que venham a contribuir com os conhecimentos já descritos na literatura especializada no assunto. Além disso, são indispensáveis para proporcionar o avanço da área e para descrever, com maior exatidão, os processos de aprendizagem durante o envelhecimento.

\section{Referências bibliográficas}

ABREU, Izabella Dutra de; FORLENZA, Orestes Vicente; BARROS, Hélio Lauar de. Demência de Alzheimer: correlação entre memória e autonomia. Rev Psiq Clin., São Paulo, v. 32 n. 3, p.131-136, 2005.

ALMEIDA, Osvaldo. Queixa de problemas com a memória e o diagnóstico de demência. Arq. Neuro-Psiquiatr., São Paulo, v. 56, n.3A, p. 412-418, set.1998.

. Instrumentos para Avaliação de Pacientes com Demência. Rev. Psiq. Clin., São Paulo, v. 26, n.2, Edição Especial, 1999.

AMERICAN PSYCHIATRY ASSOCIATION. Diagnostic and Statistical Manual of Mental Disorders, $4^{\text {th }}$. ed. Washington, D.C.: APA, 1994. 
ANDERY, Maria Amélia. Especificidades e implicações da interpretação da linguagem como comportamento verbal. In: TOURINHO, E.Z.; LUNA, S.V. Análise do comportamento: investigações históricas, conceituais e aplicadas. São Paulo: Editora Roca, 2010. p.61-99,

ANDRADE, Sancler Lopes. Vida prática e reabilitação neuropsicológica. In: FUENTES, Daniel; MALLOY-DINIZ, Leandro; CAMARGO, Cândida Pires; COSENZA, Ramon Moreira. Neuropsicologia: teoria e prática. Porto Alegre: Artmed, 2008. p.381-398.

ARGIMON, Irani; STEIN, Liliam. Habilidades cognitivas em indivíduos muito idosos: um estudo longitudinal. Cad. Saúde Pública, Rio de Janeiro, v. 21, n.1, p. 64-72, jan-fev. 2005.

ÁVILA, Renata; BOTTINO, Cassio Machado de Campos. Avaliação neuropsicológica das demências. In: MALLOY-DINIZ, Leandro; CAMARGO, Cândida Pires; COSENZA, Ramon Moreira Neuropsicologia: teoria e prática. Porto Alegre: Artmed, 2008. p.364-380

BARROS, Romariz da Silva. Uma introdução ao comportamento verbal. Rev. Bras. Ter. Comp. Cogn., São Paulo, v.5, n.1, p. 73-82, 2003.

BENNETT, David; WILSON, Robert; SCHNEIDER, Julie et al. Natural history of mild cognitive impairment in older persons. Neurology, [S.1], v. 59, n.2, p. 198-205, jul.2002.

BODI, Nikolett; CSIBRI, Eva; MYERS, Catherine. et al Associative learning, asquired equivalence, and flexible generalization of knowledge in mild Alzheimer disease. Cog Behav Neurol., [S.1.], v. 22, n.2, p. 89-94, jun. 2009.

BONINI, Daniela. O papel da família na reabilitação do paciente afásico. 1998, Monografia (Especialização em Linguagem), CEFAC - Centro de Estudos em Fonoaudiologia Clínica, São Paulo, 1998.

CARAMELLI, Paulo; BARBOSA, Maira Tonidandel. Como diagnosticar as quatro causas mais frequentes de demência? Rev Bras Psiquiatr, Rio de Janeiro, v. 24, suppl.1, p. 7-10, abr. 2002.

CARAMELli, Paulo; CARTHERY, Maria Tereza; PORTO, Catarina Segreti et al. Teste de fluência verbal no diagnóstico da doença de Alzheimer leve: notas de corte em função da escolaridade. Arq. Neuro-Psiquiatr., São Paulo, v.61, suppl. 2, 2003.

CATANIA, Charles. Aprendizagem: comportamento, linguagem e cognição. $4^{\mathrm{a}}$ ed. Porto Alegre: Artmed, 1999.

CHARCHAT-FICHMAN, Helenice; CARAMELLI, Paulo; SAMESHIMA, Kiyoko; NITRINI, Ricardo. Declínio da capacidade cognitiva durante o envelhecimento. Rev Bras Psiquiatr., Rio de Janeiro, v. 27, n.12, p. 79-82, 2005.

CHAVES, Marcia; CARMOZZATO, Ana; GODINHO, Claudia et al. Validity of the clinical dementia rating scale for the detection and staging of dementia in Brazilian patients. Alzheimer Dis Assoc Disord., [S.1.] v. 21, n. 3, p. 210-217, jul-set 2007.

COSENZA, Ramon Moreira; FUENTES, Daniel; MALLOY-DINIZ, Leandro. A evolução das ideias sobre a relação entre cérebro, comportamento e cognição. In: FUENTES, Daniel; MALLOY-DINIZ, Leandro; CAMARGO, Cândida Pires; COSENZA, Ramon Moreira. Neuropsicologia: teoria e prática. Porto Alegre: Artmed, 2008. p.15-19.

CORRÊA, Antônio Carlos de Oliveira. Neuropsicologia da memória e sua avaliação. In: FUENTES, Daniel; MALLOY-DINIZ, Leandro; CAMARGO, Cândida Pires; COSENZA, Ramon Moreira. Neuropsicologia: teoria e prática. Porto Alegre: Artmed, 2008. p.168-186. 
DAMASCENO, Benito Pereira. Comprometimento cognitivo leve e doença de Alzheimer incipiente. In: CAIXETA, L. Demência: abordagem multidisciplinar. São Paulo: Editora Atheneu, 2006. p.129-140

EPEL, Ellisa. Telomeres in a life-span perspective: a new Psychobiomarker? Current Directions in Psychological Science, [S.1.], v.18, n.1, p. 6-10, febr.2009.

EPEL, Ellisa; BLACKBURN, Elizabeth.; LIN, Jie. et al. Accelerated telomere shorting in response to life stress. PNAS, [S.1.] v. 10, n. 49, p. 17312-15, 2008.

FOLSTEIN, Marshal; FOLSTEIN, Susan; MCHUGH, Paul. Mini Mental state. A practical method for grading the cognitive state of patients for the clinician. J Psychiatr Res.,[S.1.], v.12, n.3, p.189-98, nov.1975.

HUGHES, Charles; BERG, Leonard; DANZIGER, Warren et al. A New Clinical Scale for the Staging of Dementia. Brit. J. Psychiat.[S.1.], v. 140, p. 566-572, jun.1982.

JACK, Clifford; PETERSEN, Ronald; XU, Yuanyuan et al. Rates of hippocampal atrophy correlate with change in clinical status in aging and AD. Neurology, [S.1.] v.55, n. 4, p. 484-489, aug. 2000.

KALACHE, Alexandre; VERAS, Renato P.; RAMOS, Luiz Roberto. O Envelhecimento da População Mundial. Um Desafio Novo. Rev. Saúde Pública, São Paulo, v. 21, n. 3, p. 200-10, jun. 1987.

LUDERS, Selenita Lia Alfonso; STORANI, Maria Silvia Barros. Demência: impacto para a família e a sociedade. In: PAPALÉO-NETTO, Matheus. Gerontologia: a velhice e o envelhecimento na visão globalizada. São Paulo: Edit. Atheneu, 1996. p.146-159,

MACEDO, Elizeu Coutinho; BOGGIO, Paulo Sérgio. Novas tecnologias para reabilitação neuropsicológica. In: FUENTES, Daniel; MALLOY-DINIZ, Leandro; CAMARGO, Cândida Pires; COSENZA, Ramon Moreira. Neuropsicologia: teoria e prática. Porto Alegre: Artmed, 2008. p.399-410.

MAC-KAY, Ana Paula Machado Goyano. Comunicação, fala e linguagem nas demências. São Paulo: CEFAC. 2003.

MAIA, Alberto Luiz G.; GODINHO, Claudia; FERREIRA, Eduardo Daura et al. Aplicação da versão brasileira da escala de avaliação clínica da demência (Clinical Dementia Rating - CDR) em amostras de pacientes com demência. Arq. Neuro-Psiquiatr., São Paulo, v. 64, n.2-B, p. 485-489, jun. 2006.

MANSUR, Letícia Lessa; CARTHERY, Maria Teresa; CARAMELLI, Paulo; NITRINI, Ricardo. Linguagem e cognição na doença de Alzheimer. Psicologia: reflexão e crítica, Porto Alegre, v.18, n 3, p. 300-307, set-dez. 2005.

MARTIN, Gary; PEAR, Joseph. Modificação de comportamento: o que é e como fazer. $8^{\mathrm{a}}$ ed., São Paulo: Ed. Roca, 2009.

MATOS, Maria Amélia. As categorias formais de comportamento verbal em Skinner. In: REUNIÃO ANUAL DA SOCIEDADE DE PSICOLOGIA, 21, 1991. Anais Avaliação e Perspectivas em Psicologia. Ribeirão Preto, SP, 1991.

MCKHANN, Guy; DRACHMANN, David; FOLSTEIN, Marshall et al. Clinical Diagnosis of Alzheimer's Disease: report of the NINCDS-ADRDA work group under the auspices of Department of Health and Human Services Task Force on Alzheimer's Disease. Neurology, [S.1.], v. 34, n.7, p. 939-94, jul.1984. 
MENDEZ, Mario; ALA, Thomas; UNDERWOOD, Kathryn. Development of Scoring Criteria for the Clock Drawing Task in Alzheimer's Disease. J Am Geriatr Soc,[S.l.], v. 40, n.11, p. 1095-1099, nov.1992.

NERI, Anita Liberalesso. Contribuições da psicologia ao estudo e à intervenção no campo da velhice. RBECH, Passo Fundo, v.1, n.1, p. 69-80, jan-jun.2004.

Qualidade de vida na velhice e subjetividade. In: NERI, Anita Liberalesso (Org.) Qualidade de vida na velhice: enfoque multidisciplinar. Campinas, SP: Edit. Alínea, 2007. p. 13-62

ORGANIZAÇÃO MUNDIAL DA SAÚDE. Classificação de transtornos mentais e de comportamento da CID-10. Porto Alegre: Artes Médicas, 1992.

PERLMUTTER, Marion; HALL, Elizabeth. Adult development and aging. $2^{\text {nd }}$ ed. New York, Publisher: Wiley; 1992.

PFEFFER, Raphael; KUROSAKI, Tomohiro; HARRAH, Junior; et al. Measurement of Functional Activities in Older Adults in the Community. J. Gerontol., [S.1.], v. 37, n.3, p. 323-329, may 1982.

PONTES, Lívia Maria Martins; HÜBNER, Maria Martha Costa A reabilitação neuropsicológica sob a ótica da psicologia comportamental. Rev. Psiq. Clín., São Paulo, v. 35, n1, p. 6-12, 2008.

PRESTES, Valéria Menezes. Afasia e plasticidade cerebral. 1998. Monografia. (Especialização em Linguagem). CEFAC - Centro de Estudos em Fonoaudiologia Clínica, São Paulo,1998.

PROVENCHER, Veronique; BIER, Nathalie; AUDET, Therese et al. Long-term effect of a cognitive intervention on learning and participation in a significant leisure activity in early dementia of Alzheimer type: a case study. Psychol Neuropsychiatr Vieil, [S.1.], v. 7, n.2, p. 131-140, jun. 2009.

RADANOVIC, Marcia; CARTHERY-GOULART, Maria Teresa; CHARCHAT-FICHMAN, Helenice et al. Analysis of brief language tests in the detection of cognitive decline and dementia. Dementia \& Neuropsychologia, [S.1.], v.1, n. 1, p. 37-45, jan-mar. 2007.

RIBEIRO, Priscila Cristina Correa; YASSUDA, Mônica Sanches. Cognição, estilo de vida e qualidade de vida na velhice. In: NERI, Anita Liberalesso (Org.) Qualidade de vida na velhice: enfoque multidisciplinar. Campinas, SP: Edit. Alínea, 2007. p. 189-204

ROTH; Michael; MOUNTJOY Christopher et al. CAMDEX. A standardized instrument for the diagnosis of mental disorder in the elderly with special reference to the early detection of dementia. Br J Psychiatry, [S.1.], 149, p. 698-709, dec.1986.

SCAZUFCA, Marcia; CERQUEIRA, Ana Teresa Ramos; MENEZES, Paulo Rossi et al. Investigações Epidemiológicas sobre Demência nos Países em Desenvolvimento. Rev. Saúde Pública, São Paulo, v. 36, n. 6, p. 773-778, 2002.

SÉRIO, Tereza Maria de Azevedo; ANDERY, Maria Amélia; GIOIA, Paula Susana. Os conceitos de discriminação e generalização. In. Controle de estímulos e comportamento operante: uma nova introdução. São Paulo: Educ, 2008, p. 7-26,

.Comportamento verbal. In: Controle de estímulos e comportamento operante: uma nova introdução. São Paulo: Educ, 2008. p 127-152.

SÉRIO, Tereza Maria de Azevedo; MICHELETTO, Nilza; ANDERY, Maria Amália. Definição de comportamento. In: ANDERY, Maria Amália; SÉRIO, Tereza Maria de Azevedo; MICHE- 
LETTO, Nilza. Comportamento e casualidade. Laboratório de Psicologia Experimental - Programa de Estudos Pós-graduação em Psicologia Experimental, São Paulo: PUC-SP, 2009. p. $1-9$.

SILVA, David; DAMASCENO, Benito Pereira. Demência na População de Pacientes do Hospital das Clínicas da Unicamp. Arq. Neuro-Psiquiatr, São Paulo, v. 60, n.4, p. 996-999, dez.2002.

STERLIN, Carla; JOHNSON, David; TAYLOR, William et al. Associative learning over trials activates the hippocampus in healthy elderly but not mild cognitive impairment. Neuropsychol Dev Cogn B Aging Neuropsychol Cogn, [S.1.], v.15, n.2, p. 129-145, mar. 2008.

TAVARES, Darlene Mara dos Santos; RODRIGUES, Rosalina. Educação conscientizadora do idoso diabético: uma proposta de intervenção do enfermeiro. Rev Esc Enferm USP, São Paulo, v. 36, n.1, p. 88-96, mar. 2002.

TEIXEIRA, Antônio Lúcio; CARAMELLI, Paulo. Neuropsicologia das demências. In: FUENTES, Daniel; MALlOY-DINIZ, Leandro; CAMARGO, Cândida Pires; COSENZA, Ramon Moreira. Neuropsicologia: teoria e prática. Porto Alegre: Artmed, 2008. p.356-363,

YASSUDA, Mônica Sanches. Memória e envelhecimento saudável. In: FREITAS, Elizabete Viana de; PY, Ligia et al. Tratado de Geriatria e Gerontologia. 3ª.ed.Rio de Janeiro-RJ, Editora Guanabara Koogan, 2002. p. 914-920.

YESAVAGE, Jerome Albert; BRINK, Thore; ROSE, Timothy et al. Development and validation of geriatric depression screening scale: a preliminary report. J Psychiatric Res., [S.1.], v.17, n.1, p. 37-49, 1982-1983.

WANG, Hong; KARP, Angela; HERLITZ, Anna et al. Personality and lifestyles in relation to dementia incidence. Neurology, [S.1.], v. 72, n.3, p. 253-259, jan.2009.

YESAVAGE, J.A.; BRINK, T.L.; ROSE, T.L. et al. Development and validation of geriatric depression screening scale: a preliminary report. J Psychiatric Res., [S.1.], v.17, n.1, p. 37-49, 1982-1983.

WANG, H.X.; KARP, A.; HERLITZ, A. et al. Personality and lifestyles in relation to dementia incidence. Neurology, [S.1.], v. 72, n.3, p. 253-259, jan.2009.

Recebido em: 22/04/2013

Aceite em: 25/08/2013

Juliana F. Cecato é psicóloga clínica, especialização em psicopedagogia e mestre em Ciências da Saúde pela Faculdade de Medicina de Jundiaí (FMJ). Atualmente leciona na FMJ como professora colaboradora. Endereço para correspondência: Rua

Prudente de Moraes, 111. Jundiaí/SP, Brasil. CEP 13201-004 E-mail: cecatojuliana@hotmail.com

José Eduardo Martinelli é geriatra, médico responsável pela disciplina de geriatria e gerontologia da Faculdade de Medicina de Jundiaí.

E-mail: drmartinelli@terra.com.br 
Luana L. Bartholomeu é psicóloga clínica e participa dos grupos de pesquisa do Centro Universitário FIEO. E-mail: luanasilvaluz@gmail.com

Patricia P. Ferreira é psicóloga clínica e participa dos grupos de pesquisa do Centro Universitário FIEO. E-mail: patricia.psico@outlook.com

José Maria Montiel é psicólogo clínico e professor do Centro Universitário FIEO. E-mail: montieljm@hotmail.com

Daniel Bartholomeu é psicólogo clínico e professor do Centro Universitário FIEO. E-mail: $\underline{\mathrm{d} \text { bartholomeu@yahoo.com.br }}$ 